\title{
ORDER OF THE RUNGE-KUTTA METHOD AND EVOLUTION OF THE STABILITY REGION ${ }^{1}$
}

\author{
Hippolyte Séka ${ }^{\dagger}$, Kouassi Richard Assui ${ }^{\dagger \dagger}$ \\ Institut National Polytechnique Houphouët-Boigny, \\ BP 1093 Yamoussoukro, Côte d'Ivoire \\ †hippolyte.seka@inphb.ci, ${ }^{\dagger}$ r.assui@yahoo.fr
}

\begin{abstract}
In this article, we demonstrate through specific examples that the evolution of the size of the absolute stability regions of Runge-Kutta methods for ordinary differential equation does not depend on the order of methods.
\end{abstract}

Keywords: Stability region, Runge-Kutta methods, Ordinary differential equations, Order of methods.

\section{Introduction}

Representations of the stability regions of Runge-Kutta methods are presented in several literatures $[1-8,11,13]$. It has been found that the stability region varies according to the order of the method. However, it is not proven in the literature whether or not there is a relation between the evolution of the size of the region of stability and the order of the method. In this article, we demonstrate that the evolution of the size of the stability region does not depend on the order of the methods. For that we exhibit methods whose regions of stability grow according to the order. Subsequently, we give a counter-example where we introduce a new 8 order method [12]. We compare the stability region of this new 8 order method with those of certain lower order methods. We show that the stability regions of lower order methods are larger than that of the new 8 order method. The study will be done in accordance with the following plan: in Section 2 we describe some generalities on the stability regions, in Section 3 we present some stability functions, in Section 4 we present the new 8 order method and its stability regions, Section 5 we give a conclusion.

\section{Generalities on the stability regions}

Consider a general form of the first-order ODE given below:

$$
y^{\prime}=f(x, y(x))
$$

with the initial condition $y\left(x_{0}\right)=y_{0}$ for the interval $x_{0} \leq x \leq x_{n}$. Here, $x$ is the independent variable, $y$ is the dependent variable, $n$ is the number of point values, and $f$ is the function of the derivation. The goal is to determine the unknown function $y(x)$ whose derivative satisfies (1.1) and the corresponding initial values. In doing so, let us discretize the interval $x_{0} \leq x \leq x_{n}$ to be

$$
x_{0}, \quad x_{1}=x_{0}+h, \quad x_{2}=x_{0}+2 h, \ldots, \quad x_{n}=x_{0}+n h,
$$

\footnotetext{
${ }^{1}$ We would like to express our deepest appreciation and gratitude to Professor Sergey Khashin of Ivanovo State University who provided us the possibility to coordinate and complete this article.
} 
where $h$ is the fixed step size. With the initial condition $y\left(x_{0}\right)=y_{0}$, the unknown grid function $y_{1}, y_{2}, y_{3}, \cdots, y_{n}$ can be calculated by using the Runge-Kutta method of the order 8 (RK8 method).

The 8 -th order method is thus obtained by the resolution of the 200 equations with 11 stages [12] on Maple.

Lets consider the Butcher tableau of 8 order and 11 steps RK method (see Fig. 1):

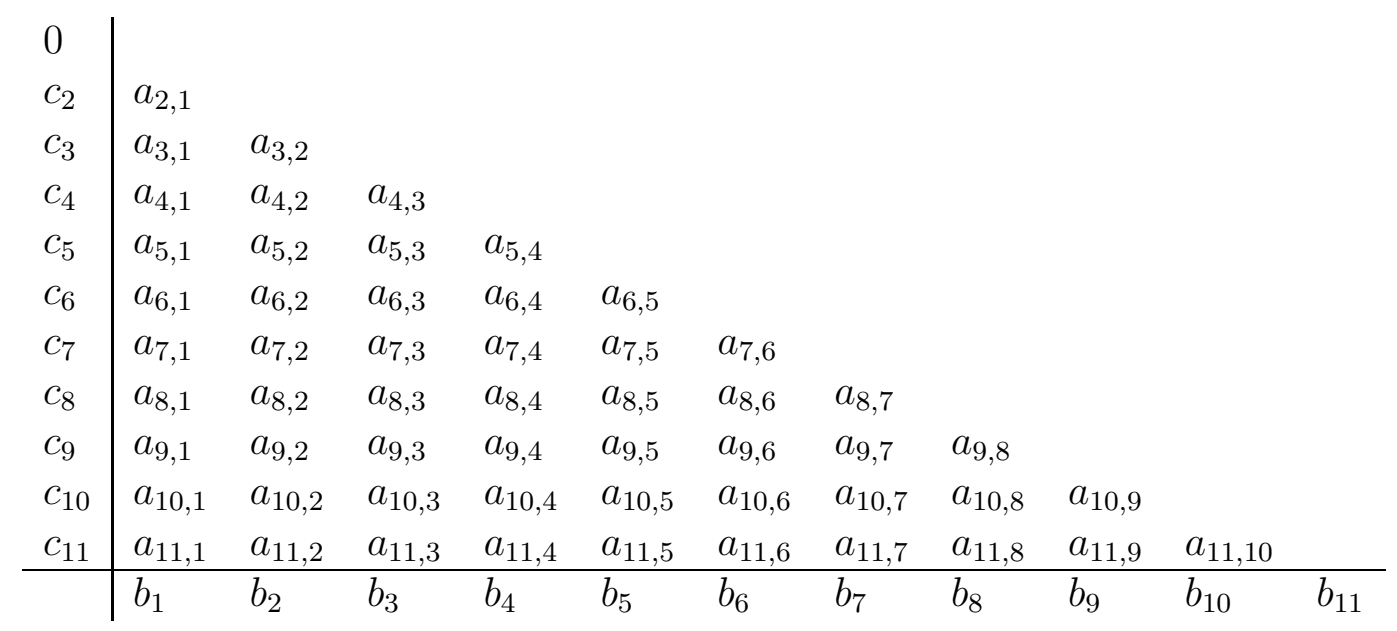

Figure 1. Butcher tableau of RK8 method.

The numerical solution is given by the formula

$$
y_{i+1}=y_{i}+h\left(\sum_{s=1}^{11} b_{s} k_{s}\right)
$$

with

$$
k_{s}=f\left(x_{i}+c_{s} h, y_{i}+h \sum_{j=1}^{s-1} a_{s, j} k_{j}\right), \quad x_{i+1}=x_{i}+h .
$$

The concept of absolute stability, in its simplest form, is based on the analysis of the behavior, according to the values of the step $h$, of the numerical solutions of the model equation [9-12]:

$$
u^{\prime}(t)=\lambda u(t) .
$$

Using (1.3) and (1.4), we obtain:

$$
\text { for } s \geq 1, \quad k_{s}=\lambda\left(y_{i}+h \sum_{j=1}^{s-1} a_{s, j} k_{j}\right)
$$

which gives:

$$
y_{i+1}=\zeta(h \lambda) y_{i} .
$$

If $z=h \lambda$, then the absolute stability region is the set

$$
\{z \in \mathbb{C}|| \zeta(z) \mid \leq 1\} .
$$




\section{Presentation of some stability functions}

Consider the standard Runge-Kutta methods of orders 1 to 4 . When (1.2) and (1.3) are applied to the model problem (1.4), the resulting equations are

$$
\begin{array}{lc}
\text { RK1: } & y_{i+1}=(1+z) y_{i} ; \\
\text { RK2: } & y_{i+1}=\left(1+z+\frac{z^{2}}{2}\right) y_{i} ; \\
\text { RK3: } & y_{i+1}=\left(1+z+\frac{z^{2}}{2}+\frac{z^{3}}{6}\right) y_{i} ; \\
\text { RK4: } & y_{i+1}=\left(1+z+\frac{z^{2}}{2}+\frac{z^{3}}{6}+\frac{z^{4}}{24}\right) y_{i} .
\end{array}
$$

The stability regions are shown at the next figure:

RK1

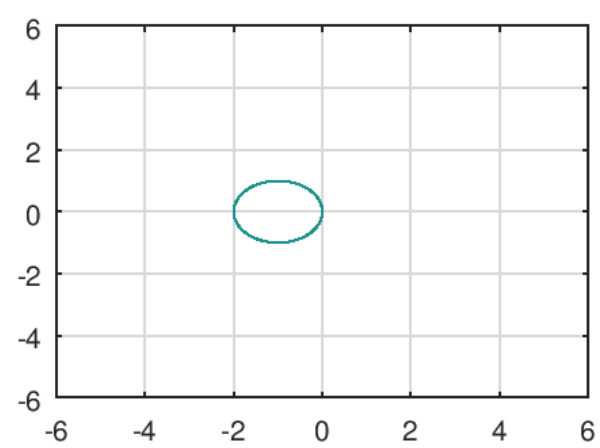

RK3

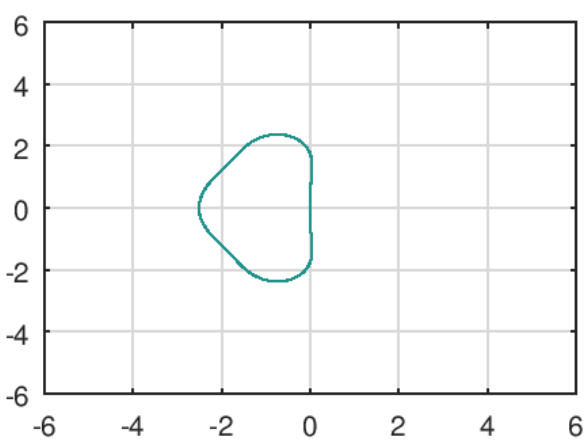

RK2

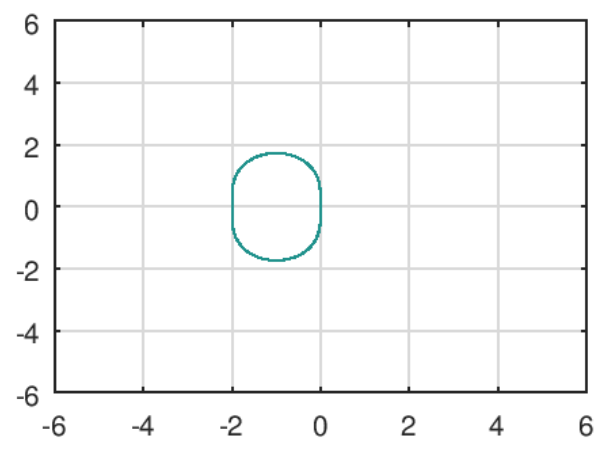

RK4

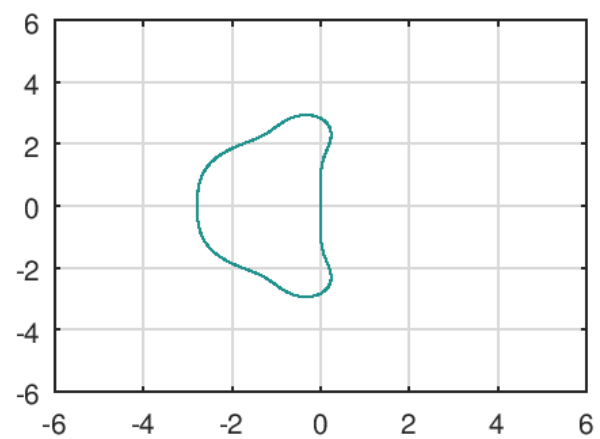

Figure 2. Evolution of the stability region according to the order.

We can see the evolution of the size of the region of stability as the order of the method increases. Let's now give a counterexample for which the stability region is very small. 


\section{Presentation of the new 8 order method and its stability regions}

The family of the $8^{\text {th }}$ order method is thus obtained by the resolution of the 200 equations with 11 stages [12] on Maple. This method depends on free parameters $b_{8}$ and $a_{10,5}$ [12].

Some of related coefficients have fixed values, not depending on $b_{8}$ and $a_{10,5}$, these coefficients are:

$$
\begin{gathered}
b_{1}=\frac{1}{20} ; \quad b_{2}=0 ; \quad b_{3}=0 ; \quad b_{4}=0 ; \quad b_{5}=0 ; \quad b_{6}=0 ; \quad b_{9}=\frac{16}{45} ; \quad b_{10}=\frac{49}{180} ; \quad b_{11}=\frac{1}{20} ; \\
c_{2}=\frac{1}{2} ; \quad c_{3}=\frac{1}{2} ; \quad c_{4}=\frac{7+\sqrt{21}}{14} ; \quad c_{5}=\frac{7+\sqrt{21}}{14} ; \quad c_{6}=\frac{1}{2} ; \\
c_{7}=\frac{7-\sqrt{21}}{14} ; \quad c_{8}=\frac{7-\sqrt{21}}{14} ; \quad c_{9}=\frac{1}{2} ; \quad c_{10}=\frac{7+\sqrt{21}}{14} ; \quad c_{11}=1 ; \\
a_{2,1}=\frac{1}{2} ; \\
a_{3,1}=\frac{1}{4} ; \quad a_{3,2}=\frac{1}{4} ; \\
a_{5,1}=\frac{1}{7} ; \quad a_{4,2}=\frac{-7-3 \sqrt{21}}{98} ; \quad a_{4,3}=\frac{21+5 \sqrt{21}}{49} ; \\
a_{6,1}=\frac{11+\sqrt{21}}{84} ; \quad a_{5,2}=0 ; \quad a_{5,3}=\frac{4 \sqrt{21}}{63}+\frac{2}{7} ; \quad a_{5,4}=\frac{21-\sqrt{21}}{252} ; \\
a_{28} ; \quad a_{6,2}=0 ; \quad a_{6,3}=\frac{9+\sqrt{21}}{36} ; \quad a_{6,4}=\frac{-231+14 \sqrt{21}}{360} ; \quad a_{6,5}=\frac{63-7 \sqrt{21}}{80} ; \\
a_{7,1}=\frac{10-\sqrt{21}}{42} ; \quad a_{7,2}=0 ; \\
a_{11,1}=0 ; \quad a_{11,2}=0 ; \quad a_{11,9}=\frac{28-28 \sqrt{21}}{42} ; \quad a_{9,2}=0 ; \\
a_{10,1}=\frac{1}{14} ; \quad a_{10,2}=0 ; \quad a_{10,9}=\frac{4 \sqrt{21}}{35}+\frac{132}{245} ;
\end{gathered}
$$

And the others are expressed in terms of $b_{8}$ and $a_{10,5}$ :

$$
\begin{gathered}
b_{7}=-b_{8}+\frac{49}{180} ; \\
a_{7,3}=-(24 / 35) a_{10,5}-136 / 105-(12 / 245) a_{10,5} \sqrt{21}+(656 / 2205) \sqrt{21} \\
a_{7,4}=7-(3 / 10) a_{10,5} \sqrt{21}-(71 / 45) \sqrt{21}+(3 / 10) a_{10,5} ; \\
a_{7,5}=-(3 / 10) a_{10,5}+(3 / 10) a_{10,5} \sqrt{21}-43 / 6+(169 / 105) \sqrt{21} ; \\
a_{7,6}=-(277 / 735) \sqrt{21}+181 / 105+(12 / 245) a_{10,5} \sqrt{21}+(24 / 35) a_{10,5} \\
a_{8,1}=-\frac{180 b_{8} \sqrt{21}-49 \sqrt{21}-1800 b_{8}+343}{7560 b_{8}} ; \quad a_{8,2}=0 ; \\
a_{8,5}=-\frac{441 a_{10,5} \sqrt{21}-3240 a_{7,5} b_{8}-28 \sqrt{21}+882 a_{7,5}-2205 a_{10,5}+147}{3240 b_{8}} \\
a_{8,6}=\frac{72 a_{10,5} \sqrt{21}+1620 a_{7,6} b_{8}-29 \sqrt{21}-441 a_{7,6}-252 a_{10,5}+119}{1620 b_{8}}
\end{gathered}
$$


And also:

$$
\begin{aligned}
& a_{8,3}=-\frac{900 b_{8} \sqrt{21}+11340 a_{7,2} b_{8}+11340 a_{8,6} b_{8}-98 \sqrt{21}-3087 a_{7,2}-4860 b_{8}+686}{11340 b_{8}} ; \\
& a_{8,7}=\frac{49}{1620 b_{8}} \\
& a_{8,4}=\frac{\left(c_{8}^{2} / 2\right)-a_{8,2} c_{2}-a_{8,3} c_{3}-a_{8,5} c_{5}-a_{8,6} c_{6}-a_{8,7} c_{7}}{c_{4}} \\
& a_{9,3}=(1 / 8) a_{10,5} \sqrt{21}-(1 / 8) a_{10,5}-(1 / 72) \sqrt{21}+1 / 72 ; \\
& a_{9,4}=-49 / 288-(7 / 32) a_{10,5} \sqrt{21}+(7 / 288) \sqrt{21}+(49 / 32) a_{10,5} ; \\
& a_{9,5}=(7 / 32) a_{10,5} \sqrt{21}-(35 / 576) \sqrt{21}-(49 / 32) a_{10,5}+21 / 64 ; \\
& a_{9,6}=-(1 / 8) a_{10,5} \sqrt{21}+(1 / 8) a_{10,5}+(1 / 72) \sqrt{21}+5 / 36 ; \\
& a_{9,7}=91 / 576+(7 / 192) \sqrt{21}-(585 / 1568) b_{8} \sqrt{21}-(405 / 224) b_{8} ; \\
& a_{9,8}=(585 / 1568) b_{8} \sqrt{21}+(405 / 224) b_{8} ; \\
& a_{10,3}=-(6 / 49) a_{10,5} \sqrt{21}-(2 / 7) a_{10,5}+(2 / 147) \sqrt{21}+2 / 63 ; \\
& a_{10,4}=1 / 9-a_{10,5} ; \\
& a_{10,6}=(2 / 7) a_{10,5}-803 / 2205+(6 / 49) a_{10,5} \sqrt{21}-(59 / 735) \sqrt{21} ; \\
& a_{10,7}=1 / 9+(1 / 42) \sqrt{21}+(2295 / 686) b_{8}+(495 / 686) b_{8} \sqrt{21} ; \\
& a_{10,8}=-(2295 / 686) b_{8}-(495 / 686) b_{8} \sqrt{21} ; \\
& a_{11,3}=(2 / 3) a_{10,5} \sqrt{21}-(2 / 3) a_{10,5}-(2 / 27) \sqrt{21}+2 / 27 \text {; } \\
& a_{11,4}=-(7 / 6) a_{10,5} \sqrt{21}+(7 / 54) \sqrt{21}+(49 / 6) a_{10,5}-49 / 54 ; \\
& a_{11,5}=(7 / 27) \sqrt{21}-77 / 54-(49 / 6) a_{10,5}+(7 / 6) a_{10,5} \sqrt{21} ; \\
& a_{11,6}=(2 / 3) a_{10,5}-64 / 135-(2 / 3) a_{10,5} \sqrt{21}+(94 / 135) \sqrt{21} ; \\
& a_{11,7}=7 / 18-(265 / 98) b_{8} \sqrt{21}-(215 / 14) b_{8} ; \\
& a_{11,8}=(265 / 98) b_{8} \sqrt{21}+(215 / 14) b_{8} .
\end{aligned}
$$

The numerical solution is given by the formula (1.2). The values of $k_{s}$ are given by the formula (1.3). We can notice that if $b_{8}=49 / 180$ and $a_{10,5}=1 / 9$, then we find the method of Cooper-Verner [1, 12].

With the help of Maple, the stability function depends on $a_{10,5}$ and is given by [12]:

$$
\begin{gathered}
\zeta(z)=1+z+\frac{1}{2} z^{2}+\frac{1}{6} z^{3}+\frac{1}{24} z^{4}+\frac{1}{120} z^{5}+\frac{1}{720} z^{6}+\frac{1}{5040} z^{7}+\frac{1}{40320} z^{8} \\
+\left(-\frac{797}{50803200}+\frac{1}{25200} a_{10,5}+\frac{37}{4233600} \sqrt{21} a_{10,5}-\frac{499}{152409600} \sqrt{21}\right) z^{9} \\
+\left(\frac{1}{470400}+\frac{1}{2083725} \sqrt{21}-\frac{31}{940800} a_{10,5}-\frac{61}{8467200} \sqrt{21} a_{10,5}\right) z^{10} \\
+\left(-\frac{1}{29030400}-\frac{13}{4267468800} \sqrt{21}+\frac{11}{1612800} a_{10,5}+\frac{353}{237081600} \sqrt{21} a_{10,5}\right) z^{11} .
\end{gathered}
$$


For $a_{10,5}=10^{6}$ we find

$$
\begin{aligned}
& \zeta(z)=1+z+\frac{1}{2} z^{2}+\frac{1}{6} z^{3}+\frac{1}{24} z^{4}+\frac{1}{120} z^{5}+\frac{1}{720} z^{6}+\frac{1}{5040} z^{7} \\
& +\frac{1}{40320} z^{8}+\frac{2015999203}{50803200} z^{9}-\frac{15499999}{470400} z^{10}+\frac{197999999}{29030400} z^{11} \\
& +\frac{190285643}{21772800} \sqrt{21} z^{9}-\frac{60046871}{8334900} \sqrt{21} z^{10}+\frac{6353999987}{4267468800} \sqrt{21} z^{11} .
\end{aligned}
$$

The stability region of the new RK8 method for $a_{10,5}=10^{6}$ is given by Fig. 3 .

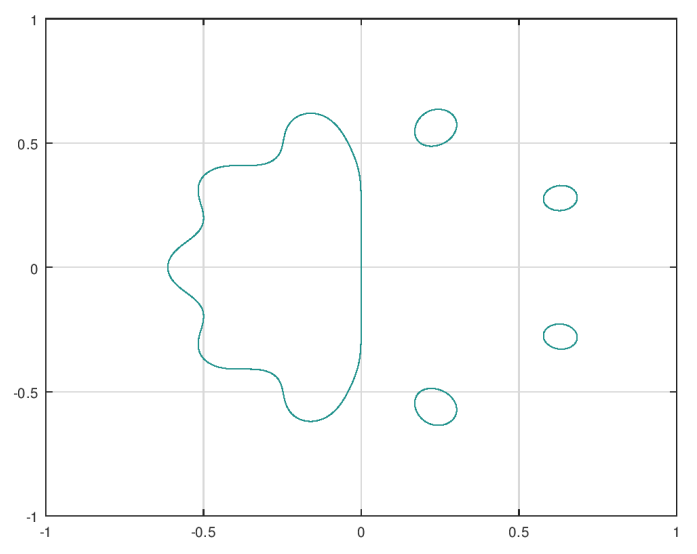

Figure 3. Stability region of the new RK8 method for $a_{10,5}=10^{6}$.

We can see that the stability region of the new method of order 8 is smaller than $2,3,4$. There is a decrease in the values of $x$ and $y$.

For $a_{10,5}=10^{12}$ the stability region is the following (see Fig. 4):

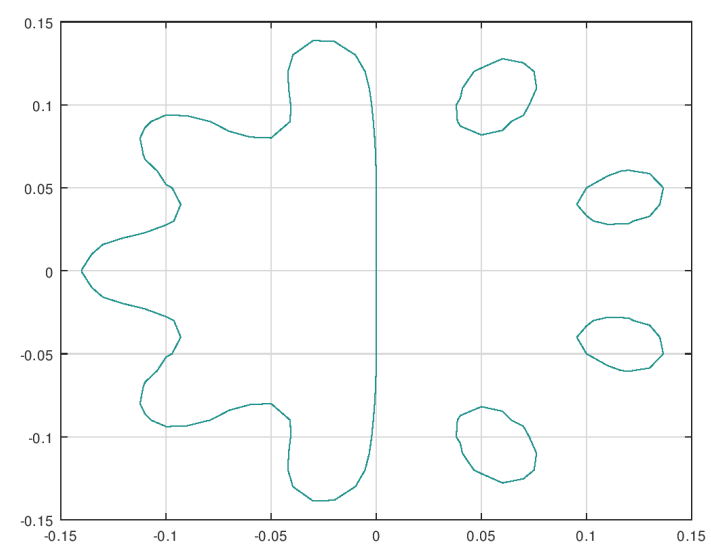

Figure 4. Stability region of the new RK8 method for $a_{10,5}=10^{12}$.

We can see that the stability region of the new method of order 8 is smaller than those of ordering regions $1,2,3,4$. There is a decrease in the values of $x$ and $y$. 
For $a_{10,5}=\underbrace{9 \ldots 9}_{37 \text { times }}$ the stability region is is shown at the next figure:

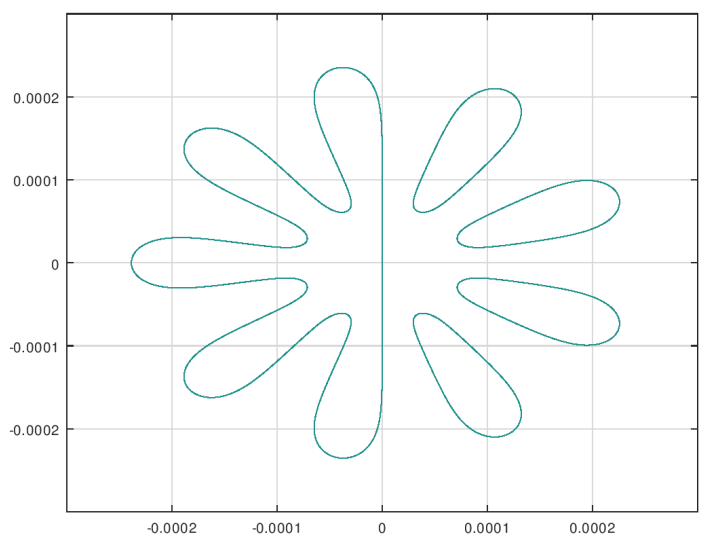

Figure 5. Stability region of the new RK8 method.

We find that the values of $x$ and $y$ have very strongly diminished and the region of stability is very small.

\section{Conclusion}

Presumably, by representing the domains of stability of methods of the order of $1,2,3,4$, one could assume that the higher the order, the greater the area of stability. However, a new 8 order method is discovered. The stability region of this 8 order method is smaller than that of the regions of orders $2,3,4$. We can therefore conclude that the evolution of the size of the stability regions of Runge-Kutta methods does not depend on the order of the method.

\section{REFERENCES}

1. Butcher J.-C. Numerical Methods for Ordinary Differential Equations. 2nd ed. John Wiley \& Sons Ltd., 2008. 175 p. DOI: $10.1002 / 9780470753767$

2. Calvo M., Montijano J. I., Randez L. A new embedded pair of Runge-Kutta formulas of orders 5 and 6. Comput. Math. Appl., 1990. Vol. 20, No. 1. P. 15-24. DOI: 10.1016/0898-1221(90)90064-Q

3. Cassity C. R. The complete solution of the fifth order Runge-Kutta equations. SIAM J. Numer. Anal., 1969. Vol. 6, No. 3. P. 432-436. DOI: $10.1137 / 0706038$

4. Feagin T. A tenth-order Runge-Kutta method with error estimate. In: Proc. of the IAENG Conf. on Scientific Computing. Hong Kong, 2007. Accessible at https://sce.uhcl.edu/feagin/courses/rk10.pdf

5. Feagin T. High-Order Explicit Runge-Kutta Methods. 2013. Accessible at http://sce.uhcl.edu/rungekutta

6. Hairer E., Nørsett S.P., Wanner G. Solving Ordinary Differential Equations I. Nonstiff Problems. Springer Ser. Comput. Math., vol. 8. Berlin, Heidelberg: Springer-Verlag, 1993. 528 p. DOI: $10.1007 / 978-3-540-78862-1$

7. Houben S. Stability Regions of Runge-Kutta Methods. Eindhoven University of Technology, 2002. Accessible at https://www.win.tue.nl/casa/meetings/seminar/previous/_abstract020220_files/talk.pd

8. Jackiewicz Z. General Linear Methods for Ordinary Differential Equations. John Wiley \& Sons, Inc., 2009. 482 p. DOI: $10.1002 / 9780470522165$

9. Khashin S. I. List of Some Known Runge-Kutta Methods Family. Preliminary version. 2013. Accessible at http://math.ivanovo.ac.ru/dalgebra/Khashin/rk/sh_rk.html

10. Kashin S. I. Estimating the error in classical Runge-Kutta methods. Comput. Math. Math. Phys., 2014. Vol. 54, No. 5. P. 767-774. DOI: 10.1134/S0965542514050145 
11. Liu M.Z., Song M.H., Yang Z.W. Stability of Runge-Kutta methods in the numerical solution of equation $u^{\prime}(t)=a u(t)+a_{0} u([t])$. J. Comput. Appl. Math., 2004. Vol. 166, No. 2. P. 361-370. DOI: $10.1016 /$ j.cam.2003.04.002

12. Seka H., Assui K. R. A New Eighth Order Runge-Kutta Family Method. J. Math. Res., 2019. Vol. 11, No. 2. P. 190-199. DOI: 10.5539/jmr.v11n2p190

13. Velagala S. R. Stability Analysis of the 4th order Runge-Kutta Method in Application to Colloidal Particle Interactions. Master's thesis. University of Illinois, Urbana-Champaign, USA, 2014. Accessible at http://hdl.handle.net/2142/72750 